\title{
FDA Manufacturer Evaluation Conclusion Code Hierarchy
}

National Cancer Institute

\section{Source}

National Cancer Institute. FDA Manufacturer Evaluation Conclusion Code Hierarchy. NCI

Thesaurus. Code C91802.

Terminology that represents conclusions of an evaluation used by the CDRH with respect to reporting Medical Device events. 